\title{
Incidence of amyotrophic lateral sclerosis in Sicily: A population based study
}

\author{
PAOLO RAGONESE ${ }^{1}$, ELEONORA CELLURA ${ }^{1}$, PAOLO ARIDON ${ }^{1}$, MARCO D'AMELIO $^{1}$, \\ ROSSELLA SPATARO $^{1,2}$, ALFONSA CLAUDIA TAIELLO ${ }^{1,2}$, DAVIDE MAIMONE ${ }^{3}$, \\ VINCENZO LA BELLA ${ }^{1,2}$ \& GIOVANNI SAVETTIERI ${ }^{1}$
}

${ }^{1}$ Dipartimento di Biomedicina Sperimentale e Neuroscienze Cliniche, ${ }^{2} A L S$ Clinical Research Centre at the Department
of Experimental Biomedicine and Clinical Neurosciences, (Dipartimento di Biomedicina Sperimentale e Neuroscienze
Cliniche), University of Palermo, and ${ }^{3}$ Centro di riferimento regionale per le Malattie Neuroimmunologiche, UOC di
Neurologia, Azienda Ospedaliera Garibaldi, Catania, Italy

\begin{abstract}
Our objective was to investigate incidence of amyotrophic lateral sclerosis (ALS) in Sicily, southern Italy, by means of a population based study. We included people with ALS resident in five Sicilian provinces, whose onset occurred in the two-year period 2005-2006 (population at 31 December 2006: 3,481,096 inhabitants). A multisource case-finding procedure was adopted and patients were classified as affected by ALS according to revised El Escorial criteria. During the two-year surveillance period, 97 patients meeting eligibility criteria included 57 males (58.8\%) and 40 females (41.2\%). Crude annual incidence rate was $1.4 / 100,000$ person years (95\% CI $1.33-1.47)$. The incidence rate was higher in males $(1.71 / 100,000$; CI 1.61-1.81) than in females (1.11/100,000; CI 1.01-1.21). Standardized incidence rate for the total population in the 45-74-years-old age group was 3.22 (CI 3.11-3.33). Prevalence rate was 6.0/100,000 (CI 5.97-6.03), higher in males (7.1/100,000; CI 7.02-7.18) than females (4.9/100,000; CI 4.86-4.94). In conclusion, ALS rates observed in the present study are higher in males than females, with a peak of incidence at 70 years of age in both genders. These findings are consistent with those of other population based European studies.
\end{abstract}

Key words: Amyotrophic lateral sclerosis, epidemiology, incidence, population based, Sicily

\section{Introduction}

Amyotrophic lateral sclerosis (ALS) is a neurodegenerative disorder with an incidence range between 1.5 and 2.5 cases per 100,000 person-years (1). The mean survival time from onset of symptoms is approximately two years (1). Incidence based studies are expensive, and mortality studies have been used to obtain indirect estimates of ALS frequency $(2-4)$. More recently, several studies have suggested that the validity of this methodology is questionable and several pitfalls may challenge the accuracy of official statistics data $(5,6)$. Previous surveys performed in Italy indicated mean annual incidence rates ranging from 2.5 in Piedmont and Valle d'Aosta region, in northern Italy (7), to 1.6 in Apulia region, in southern
Italy (8). Reported prevalence ranges from 1.56 observed in Sardinia (9) to 5.4 in Reggio Emilia (10) per 100,000 population. There is currently limited updated data on ALS frequency in southern Italy and in Sicily in particular, to offer the possibility of geographical comparison. We performed a survey in Sicily, which aimed to clarify the epidemiological and clinical features of ALS and to compare these data with those from other studies previously performed.

\section{Methods}

This study was conducted in Sicily, an island in southern Italy with nine administrative districts,

The first two authors gave an equal contribute to the project.

Correspondence: Paolo Ragonese, Dipartimento di Biomedicina Sperimentale e Neuroscienze Cliniche, Università di Palermo, Via G. La Loggia, 1-90129-Palermo, Italy. Fax: 39 0916555152; E-mail: paolo.ragonese@unipa.it 
called provinces. The area covers 25,710 square kilometres. The population of the study area was $5,016,861$ based on the Italian Statistics Bureau (ISTAT) data for the year 2006. In Sicily there are 35 neurological departments and three referral centres for ALS, located at the neurological departments of the university hospitals of Palermo, Catania and Messina. We studied ALS in five provinces (Agrigento, Caltanissetta, Catania, Palermo, Trapani), having previously ascertained that there was an adequate participation rate from physicians and from neurological departments in these provinces. The population of the five provinces considered was 3,481,096 inhabitants on 31 December 2006. The primary sources we targeted included neurological departments located in the study area and hospital discharge diagnosis data bank (HDDB). The search code used for diagnosis of ALS was 335.2 (International Classification of Disease, 10th revision). The regional registry for riluzole prescriptions was also used to trace patients who were subsequently interviewed and examined personally. Standardized face-to-face questionnaires with clinical information were used for this purpose. All of the forms were reviewed by neurologists expert in the field, who confirmed the diagnoses and decided for patients' inclusion. ALS diagnoses were based on the revised version of the El Escorial criteria (11). These criteria distinguish four levels of diagnostic certainty: definite, probable, probable laboratory supported, and possible ALS. Cases with progressive muscular atrophy (PMA), primary lateral sclerosis (PLS), and progressive bulbar palsy (PBP) were included in the study and were reclassified according to the El Escorial criteria. We calculated crude, and age- and gender-specific incidence curves for patients whose onset occurred in the two-year period 2005-2006 (population at 31 December 2006: 3,481,096 inhabitants). Confidence limits (95\% CI) for incidence rates were calculated assuming a normal distribution. The study is retrospective because case finding procedure was performed by analyses of all charts retrieved at the sources used for the study. All people who experienced their first symptoms during the study period were included in the incident cohort; those who died were not considered as prevalent cases unless death occurred before prevalence day. Comparison with other studies was possible for the age interval between 45 and 74 years. Standardized rates were also calculated using the direct method for this age group, with the 1990 U.S. population as a standard, to allow comparisons with previous studies (12). Since some underestimation was possible due to the methodology used, we also calculated completeness of the case-finding procedure and incidence rates according to the capture-recapture methodology by matching the records of hospital admission, the registries of ALS centres in Sicily, and the riluzole prescription registries.

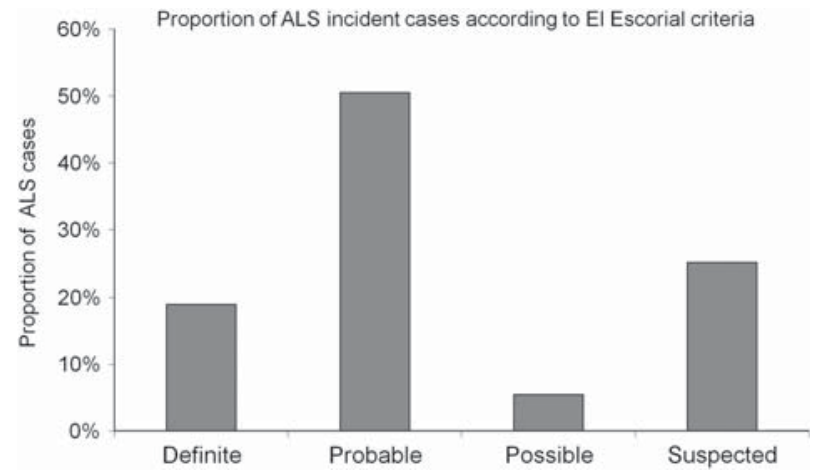

Figure 1. Proportion of ALS incident cases according to El Escorial criteria.

\section{Results}

During the two-year surveillance period, 97 patients meeting the eligibility criteria were included: 57 males $(58.8 \%)$ and 40 females $(41.2 \%)$ with a male to female ratio of $1.4: 1$. Mean age at disease onset was 58.3 years $(S D \pm 14.6)$ and mean age at diagnosis was 59.5 years. There was a similar delay between males and females in the onset of symptoms and the diagnosis (mean 15.2 months for the whole cohort; 15.6 males, 14.5 females). Twelve patients were under 45 years of age $(12.4 \%)$. No familial ALS cases were identified in the incident cohort. There were three patients with ALS-Frontal Temporal Dementia (ALS-FTD; 3.1\%), three with PLS $(3.1 \%)$ and four with PMA (4.1\%). Almost $24 \%$ of patients had bulbar onset of symptoms and $70 \%$ of individuals had spinal onset. One patient $(0.9 \%)$ experienced respiratory symptoms at onset while in $4.5 \%$ the type of onset was of uncertain classification. A higher rate of bulbar symptoms was noted among females $(31.9 \%)$ than males $(20.7 \%)$. Using the revised El Escorial diagnostic criteria, $69.4 \%$ of cases were classified as definite or probable, $5.4 \%$ were classified as laboratory supported, and $25.2 \%$ as possible (Figure 1). Crude

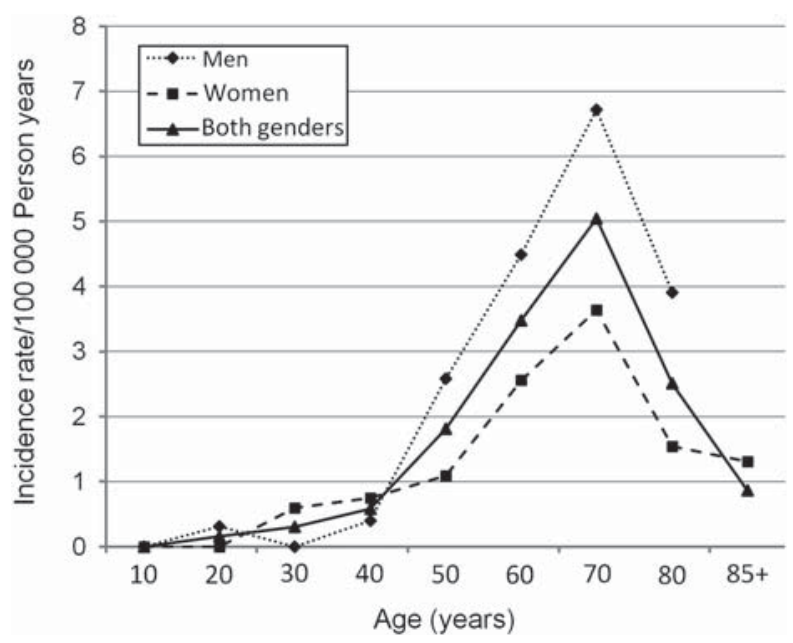

Figure 2. Crude and age- and gender-specific incidence rates of ALS in Sicily. 
Table I. Annual gender- and age-specific incidence rates of amyotrophic lateral sclerosis in Sicily, southern Italy.

\begin{tabular}{|c|c|c|c|c|c|c|c|c|c|c|c|c|}
\hline \multirow[b]{2}{*}{ Age (years) } & \multicolumn{4}{|c|}{ Males } & \multicolumn{4}{|c|}{ Females } & \multicolumn{4}{|c|}{ Both genders } \\
\hline & Population & Cases & Rate & $95 \% \mathrm{CI}$ & Population & Cases & Rate & $95 \% \mathrm{CI}$ & Population & Cases & Rate & $95 \%$ CI \\
\hline $0-17$ & $369,019.5$ & 0 & - & - & $350,100.5$ & 0 & - & - & 719,120 & 0 & - & - \\
\hline $18-24$ & $161,532.5$ & 1 & 0.31 & $0.03-0.65$ & $157,547.5$ & 0 & - & - & 319,080 & 1 & 0.16 & $0.09-0.40$ \\
\hline $25-34$ & 236,511 & 0 & - & - & $252,273.5$ & 3 & 0.59 & $0.33-0.86$ & $488,784.5$ & 3 & 0.31 & $0.11-0.50$ \\
\hline $35-44$ & $250,587.5$ & 2 & 0.40 & $0.13-0.67$ & 267,026 & 4 & 0.75 & $0.49-1.01$ & $517,613.5$ & 6 & 0.58 & $0.39-0.77$ \\
\hline $45-54$ & $212,902.5$ & 11 & 2.58 & $2.32-2.84$ & $229,575.5$ & 5 & 1.09 & $0.82-1.36$ & 442,478 & 16 & 1.81 & $1.62-2.00$ \\
\hline $55-64$ & 178,182 & 16 & 4.49 & $4.25-4.73$ & 195,566 & 10 & 2.56 & $2.29-2.83$ & 373,748 & 26 & 3.48 & $3.30-3.66$ \\
\hline $65-74$ & 148,850 & 20 & 6.72 & $6.51-6.92$ & 178,552 & 13 & 3.64 & $3.38-3.90$ & 327,402 & 33 & 5.04 & $4.87-5.21$ \\
\hline $75-84$ & 89,539 & 7 & 3.91 & $3.55-4.27$ & 129,894 & 4 & 1.54 & $1.19-1.89$ & 219,433 & 11 & 2.51 & $2.25-2.76$ \\
\hline $85+$ & 19,923 & 0 & & & 38,158 & 1 & 1.31 & $0.65-1.97$ & 58,081 & 1 & 0.86 & $0.31-1.41$ \\
\hline Total & $1,667,047$ & 57 & 1.71 & $1.61-1.81$ & $1,798,693$ & 40 & 1.11 & $1.01-1.21$ & $3,465,740$ & 97 & 1.40 & $1.33-1.47$ \\
\hline
\end{tabular}

Incidence rate among individuals over 18 years of age was 1.77 for the total population (CI 1.69-1.85); 2.20 (CI 2.09-2.31) in males, and 1.38 (CI 1.27-1.49) in females.

annual incidence rate for ALS at the end of the observation period (2005-2006) was $1.4 / 100,000$ person-years (95\% CI $1.33-1.47)$. Incidence rate was higher in males $(1.71$; CI $1.61-1.81)$ than females (1.11; CI 1.01-1.21). This proportion of higher incidence in males remained similar at all age groups. There was some variability among the five provinces but no significant differences were detected. As illustrated in Figure 2, incidence showed a progressive increase up to age 75 years, and declined thereafter maintaining a similar trend in both genders with higher rates in males at all ages. Incidence rates of ALS in the five provinces considered are shown in Table I. We compared incidence rates in Sicily to those from previous studies with respect to the age range between 45 and 74 years by adjusting all the rates to the U.S. 1990 general population, as illustrated in Table II. Adjusted incidence rates for these age groups were $3.22 / 100,000$ person-years $(95 \%$ CI $3.11-3.33)$ in both genders: 4.3 in males (CI 4.13-4.41) and 2.27 in females (CI 2.11-2.43). We also calculated the prevalence rate of ALS on 31 December 2006. Crude prevalence was 6.0 per 100,000 population (CI 5.97-6.03): 7.1 in males (95\% CI: 7.02-7.18), and 4.9 in females (CI: 4.86-4.94). By matching the three main sources of information according to the capture-recapture methodology, we estimated a rate of accuracy of our search strategy of $72.1 \%$. Recalculating incidence rates according to these calculations would give an incidence rate of 1.95 per 100,000 population for the whole population considered (95\% CI 1.88-2.02) and of 2.46 per
100,000 population for the population aged over 18 years (CI 2.39-2.53).

\section{Discussion}

Our study, performed in a southern Mediterranean island, used a retrospective cohort design to ascertain the incidence of ALS according to diagnostic criteria adopted by several previous studies $(13-15)$. The observed incidence rates were higher for males compared to females and showed a similar progressive increase up to 70 years of age in both genders, with a rapid decrease thereafter. Incidence rates observed in the present study are lower compared to those previously reported in Italy $(1,7,8,13)$. This may be due at least in part to the retrospective nature of the study. To minimize a possible underestimation we focused our study on the health districts of Sicily with high participation rates by physicians and neurologists, and where it was possible to confirm each diagnosis by direct examination of patients and of clinical records. A previous report from a single Sicilian province has shown rates lower than expected and lower than those reported in other geographic areas (16). The reported annual incidence in that study was $0.44 / 100,000$ inhabitants. It is likely that methodological differences may have influenced the results. At the time of the first study, performed in the province of Palermo, there were no disease archives and little information available for patients, particularly for rare conditions. In 1994, the El Escorial criteria were published (11), and although these criteria did not introduce specific instrumental

Table II. Standardized incidence rate in Sicily adjusted to the 1990 U.S. + population for the age groups $45-74$ years.

\begin{tabular}{lccc}
\hline Years 2005-2006 & Observed incidence rate & Adjusted incidence rate & 95\% $\mathrm{CI}^{*}$ \\
\hline Total & 3.28 & 3.22 & $3.11-3.33$ \\
Males & 4.35 & 4.27 & $4.13-4.41$ \\
Females & 2.31 & 2.27 & $2.11-2.43$ \\
\hline
\end{tabular}

*95\% CI: $95 \%$ confidence intervals for incidence rates standardized to the 1990 U.S. population for the $45-74$ years age group. 
tests, it is likely that their use may have influenced estimates of incidence. The availability of riluzole therapy has also raised the level of disease awareness among physicians and consequently, the opportunity for possible ALS patients to come to neurologists' attention. We included all four diagnostic levels of the revised version of El Escorial and included PLS and PMA in the calculation of the incidence and prevalence rates. These were classified as possible cases and accounted for a quarter of the total of ALS diagnoses. As has been previously noted, age-specific curves show a peak at about 70 years of age with a decline thereafter (17). This suggests the presence of environmental factors acting during specific critical periods of life. An alternative explanation is the possible underestimation of disease symptoms among these older individuals. Standardized rates adjusted for the 45-74 years age group as shown in Table II, make it easier to compare our results with those from previous studies performed in other geographic areas at different times $(1,17,18)$. These comparisons give further strength to the fact that the observed rates reflect the real distribution of ALS in Sicily showing incidences that are lower, but close to, those of previous reports in Italy. One possible explanation for the different rates observed in our population may relate to the different historical background of the Sicilian population that can be considered genetically admixed. A previous study has suggested that genetic admixture could account for differential risks across populations for complex degenerative diseases such as ALS $(19,20)$. However, we consider that a more probable explanation for the slightly lower incidence rates of ALS described in our study may be attributed to some underascertainment because of the retrospective data collection.

\section{Acknowledgements}

The authors thank Mario Zappia and Giovanni Majorana for their helpful collaboration. The authors also thank the anonymous referees for their contribution to a substantial revision and improvement of the study.

Declaration of interest: The authors report no conflicts of interest. The authors alone are responsible for the content and writing of the paper.

\section{References}

1. Logroscino G, Traynor BJ, Hardiman O, Chio A, Couratier P, Mitchell JD, et al. Descriptive epidemiology of amyotrophic lateral sclerosis: new evidence and unsolved issues. J Neurol Neurosurg Psychiatry. 2008;79:6-11.

2. Uccelli R, Binazzi A, Altavista P, Belli S, Comba P, Mastrantonio $\mathrm{M}$, et al. Geographic distribution of amyotrophic lateral sclerosis through motor neuron disease mortality data. Eur J Epidemiol. 2007;22:781-90.

3. Neilson S, Robinson I, Alperovitch A. Rising amyotrophic lateral sclerosis mortality in France 1968-1990: increased life expectancy and inter-disease competition as an explanation. J Neurol. 1994;241:448-55.

4. Neilson S, Gunnarsson LG, Robinson I. Rising mortality from motor neuron disease in Sweden 1961-1990: the relative role of increased population life expectancy and environmental factors. Acta Neurol Scand. 1994;90:150-9.

5. Ragonese P, Filippini G, Salemi G, Beghi E, Citterio A, D'Alessandro R, et al. Accuracy of death certificates for amyotrophic lateral sclerosis varies significantly from north to south of Italy: implications for mortality studies. Neuroepidemiology. 2004;23:73-7.

6. Marin B, Couratier P, Preux PM, Logroscino G. Can Mortality Data Be Used to Estimate Amyotrophic Lateral Sclerosis Incidence? Neuroepidemiology. 2011;36: 29-38.

7. Piemonte and Valle d'Aosta Register for Amyotrophic Lateral Sclerosis (PARALS). Amyotrophic lateral sclerosis in Italy, 1995 through 1996: a prospective analysis of incidence. Neurology. 2001;56:239-44.

8. Logroscino G, Beghi E, Zoccolella S, Palagano R, Fraddosio A, Simone IL, et al. Incidence of amyotrophic lateral sclerosis in southern Italy: a population based study. J Neurol Neurosurg Psychiatry. 2005;76:1094-8.

9. Rosati G, Pinna L, Granieri E, Aiello I, Tola R, Agnetti V, et al. Studies on epidemiological, clinical, and aetiological aspects of ALS disease in Sardinia, southern Italy. Acta Neurol Scand. 1977;55:231-44.

10. Guidetti D, Bondavalli M, Saladini R, Marcello N, Vincenti M, Colombo A. Epidemiological survey of amyotrophic lateral sclerosis in the province of Reggio Emilia (Italy). Influence of environmental exposure to lead. Neuroepidemiology. 1996;15:301-12.

11. Brooks BR, Miller RG, Swash M, Munsat TL, World Federation of Neurology Research Group on Motor Neuron Diseases. El Escorial revisited: revised criteria for diagnosis of amyotrophic lateral sclerosis. Amyotroph Lateral Scler Other Motor Neuron Disord. 2000;1:293-9.

12. Census Bureau US 1990 US http://eire.census.gav/papest/ archives/national.

13. Chiò $\mathrm{A}$, Ciccone $\mathrm{G}$, Calvo $\mathrm{A}$, Verzellino $\mathrm{M}$, Di Vito $\mathrm{N}$, Ghiglione P, et al. The Piemonte and Valle D'Aosta Register for ALS. Validity of hospital morbidity records for amyotrophic lateral sclerosis. A population based study. J Clin Epidemiol. 2002;55:723-7.

14. Traynor BJ, Codd BM, Corr B, Forde C, Frost E, Hardiman O. Incidence and prevalence of ALS in Ireland 1995-1997: a population based study. Neurology. 1999;52: 504-9.

15. Nygren I, Antonova K, Mattson P, Askmark H. The ALS/ MND prevalence in Sweden estimated by riluzole sales statistics. Acta Neurol Scand. 2005;111:180-4.

16. Salemi G, Fierro B, Arcara A, Cassata M, Castiglione MG, Savettieri G. Amyotrophic lateral sclerosis in Palermo, Italy: an epidemiological study. Ital J Neurol Sci. 1989;10: 505-9.

17. Huisman $\mathrm{MH}$, de Jong SW, van Doormaal PT, Weinreich SS, Schelhaas HJ, van der Kooi AJ, et al. Population based epidemiology of amyotrophic lateral sclerosis using capturerecapture methodology. J Neurol Neurosurg Psychiatry. 2011; May 27. (Epub ahead of print).

18. Logroscino G, Traynor BJ, HardimanO, Chiò A, Mitchell D, Swingler RJ, et al. Incidence of amyotrophic lateral sclerosis in Europe. J Neurol Neurosurg Psychiatry. 2010;81:385-90.

19. Zaldivar T, Gutierrez J, Lara G, Carbonara M, Logroscino G, Hardiman O. Reduced frequency of ALS in an ethnically mixed population: a population based mortality study. Neurology. 2009;72:1640-5.

20. Di Gaetano C, Cerutti N, Crobu F, Robino C, Inturri S, Gino S, et al. Differential Greek and northern African migrations to Sicily are supported by genetic evidence from the $Y$ chromosome. Eur J Hum Genet. 2009;17:91-9. 